\title{
Charting Sequential Person Transfers Between Devices, Agents, and Robots
}

\author{
Xiang Zhi Tan \\ zhi.tan@ri.cmu.edu \\ Carnegie Mellon University \\ Pittsburgh, PA \\ Aaron Steinfeld \\ steinfeld@cmu.edu \\ Carnegie Mellon University \\ Pittsburgh, PA
}

\author{
Michal Luria \\ mluria@cs.cmu.edu \\ Carnegie Mellon University \\ Pittsburgh, PA \\ Jodi Forlizzi \\ forlizzi@cs.cmu.edu \\ Carnegie Mellon University \\ Pittsburgh, PA
}

\begin{abstract}
In the not-so-distant future, people in service experiences are likely to interact with more than a single intelligent system, often sequentially, including different robots and devices. However, there has been sparse work exploring the characteristics of transferring people from one intelligent system to another. This paper aims to create a context-independent taxonomy to differentiate and categorize the transfer of users across robots, devices, and human staff in service interactions. We conducted two sets of design workshops where participants generated scenarios of human-multi-robot interactions and existing person transfers. Using the outcomes of both workshops, we analyzed scenarios and constructed a taxonomy for person transfers with 4-dimensions: Rationale, Type, Design, and Information Shared. We showcase different ways to utilize the taxonomy, and, through it, we discuss the trade-offs and design considerations in the implementation of person transfers.
\end{abstract}

\section{CCS CONCEPTS}

- Human-centered computing $\rightarrow$ Interaction design theory, concepts and paradigms.

\section{KEYWORDS}

design workshop, person transfers, sequential interactions

ACM Reference Format:

Xiang Zhi Tan, Michal Luria, Aaron Steinfeld, and Jodi Forlizzi. 2021. Charting Sequential Person Transfers Between Devices, Agents, and Robots. In Proceedings of the 2021 ACM/IEEE International Conference on Human-Robot Interaction (HRI '21), March 8-11, 2021, Boulder, CO, USA. ACM, New York, NY, USA, 10 pages. https://doi.org/10.1145/3434073.3444654

\section{INTRODUCTION}

Service experiences are no longer dictated by just a single device or technology, but rather by a diverse set of devices and technological advances. For instance, a person at an airport may start by checking

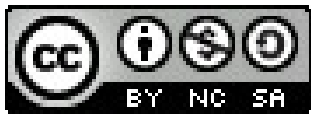

This work is licensed under a Creative Commons Attribution-NonCommercialShareAlike International 4.0 License.

HRI '21, March 8-11, 2021, Boulder, CO, USA

(C) 2021 Copyright held by the owner/author(s).

ACM ISBN 978-1-4503-8289-2/21/03.

https://doi.org/10.1145/3434073.3444654 in at the screen kiosk and dropping off their baggage. They proceed to go through security by scanning the tickets on their phones and checking for any changes to their flight plan by asking their smart voice assistant. In some places, the person may run into a guidance robot who leads them to their boarding gate [13]. Robots, like other digital interfaces that individuals encounter in this kind of environment, are not isolated singular events, but rather a part of an extended interaction involving screens, smartphones, intelligent agents, human staff, and other robots. Furthermore, existing electronic devices are slowly gaining capabilities that are making them more intelligent and interconnected. Artificial intelligence (AI) and physical embodiments are incorporated in many devices and services, blurring the lines between what is a robot, an agent, or a simple computational device. For example, ordering food has shifted from web interfaces to verbal interfaces [31], and tools like shopping carts have integrated screens and voice interfaces [32].

Concurrent with the addition of these systems is the opportunity to further streamline and improve sequential interactions within a single service experience by leveraging the connectivity and superhuman capabilities of technology. To revisit the airport example, after check-in at a kiosk, the kiosk could summon a guidance robot to lead the traveler to their boarding gate. Along the way, the guidance robot might notice that the traveler has extra time before boarding and suggest a detour to a coffee shop. It might even preorder the traveler's coffee for them. Our prior work called these sequential actions of a human interacting with one entity followed by another entity as a "transfer" [37]. Instead of using the term "robot", we choose to use the more general term "entity" to denote human staff, devices, smart agents, or robots as the transfers can happen in different combinations. As evident in our findings, the word transfer by itself is ambiguous and could mean the transfer of the person, an object (e.g., a robot transfers a suitcase to you), or information (e.g., a robot informs you of your boarding time). In this paper, we focus on person transfers - the action of a person moving from interacting with one entity to a different entity within a single service.

The design of person transfers is not straightforward, as they happen under a variety of conditions and configurations. For instance, the transfer of a customer from a kiosk to a guidance robot is different from a transfer to a human service manager. While in both cases the customer interacts with one entity and then another, the receiving entity serves different purposes and therefore requires 
different information to complete the task. We found little work addressing how to distinguish and design for different sequential actions and no coherent systematic review of this design space. Compared with simultaneous interaction with multiple devices, sequential interactions have received less attention in the literature [5], and research often focuses on immediate transfers from one system to another [38]. There is also sparse work examining how robots and smart agents fit into sequential interactions with screens, devices, and human staff members.

We aim to construct a context-independent taxonomy of the different dimensions involved in constructing a person transfer between robots and digital systems. These dimensions help us identify core factors that characterize person transfers and how they might influence the structure and design of person transfers.

To construct a taxonomy for this complex and futuristic topic, we took a design research approach. As these interactions are mostly set in the future, we lack an understanding of how to design them and the social norms around them. Design methods are well suited to tackle this gap: they allow us to extrapolate existing interactions and explore ways in which interactions may play out in the future.

We conducted two sets of design workshops with a range of experts. We first conducted a scoping workshop to explore the space of human and multiple robot (hereafter written as human-multirobot) sequential interactions. This workshop identified ways in which people might interact sequentially with multiple robots and highlighted person transfers to be one of three types of sequential interactions. We then followed with an ideation workshop that focused on explored sequential person transfers through the lens of existing person transfers between robots, devices, and human staff. This workshop yielded details on the components of person transfers, providing real-life examples which we drew on for our taxonomy. We then combined findings from the two workshops and identified emerging themes to construct a 4-dimensional taxonomy that breaks down sequential person transfers and highlights research opportunities for the future within this space.

This paper makes the following contributions:

(1) It presents a taxonomy and vocabulary to describe sequential person transfers in a way that is actionable for Human-Robot Interaction (HRI) researchers and interaction designers.

(2) It demonstrates how the different dimensions of this taxonomy can inform the interaction design of person transfers.

(3) It presents examples of how the taxonomy can be applied.

(4) It highlights potential research questions regarding person transfers.

\section{RELATED WORK}

\subsection{Multi-Device Interactions}

One of the major tenets of Human-Computer Interaction is the creation of a future where computing is ubiquitous and prevalent [42] This has led to a body of research in the area of multi-device interactions [7, 21, 23, 43]. Brudy et al. [5] developed a taxonomy to classify cross-device interactions into 6 domains: temporal, configuration, relationship, scale, dynamics, and space. They identified that the majority of the work on multi-device interaction focuses on simultaneous ("synchronous") interactions with multiple devices.
Prior research on asynchronous interactions explored how interactions can shift immediately from one device to another. Coenen et al. [8] used patterns on multiple public screens to lead users to their destinations in a train station. Ghiani et al. [11] described a system that transfers web pages from a screen to a mobile device for continuous viewing. These immediate sequential interactions have become commonplace with the deployment of commercial systems, such as Apple's Continuity, where a single action can seamlessly transition from one device to another. Ethnographic work has been done to see how they are used in real life. Raptis et al. [26] looked at online responses to Apple's Continuity feature and found that users want more control on what is presented on their device. Santosa and Wigdor [28] conducted a field study to investigate how people use multiple devices and found a need for easy information transfer and awareness between devices. More research is still needed to better understand and design interaction for sequential transfers in different contexts, especially with robotic systems.

\subsection{Sequential Service Interactions}

Sequential interactions are often modelled in service design tools such as customer journey maps or service blueprints [35]. A customer journey map documents the actions and interactions a customer may have with a service [14], and service blueprints model intended actions by user-facing and back-end workers when providing a service to the customer [4]. These techniques have been used by design researchers and user experience designers to make sense of complex interactions with technology [45]. Tonkin et al. [39] use customer journey maps as part of their methodology for designing social robotics applications. However, the findings of journey maps often focus on their specific contexts and interactions, and they do not provide much insight into the active role of transferring from one system to another throughout the journey.

\subsection{Robot(s)-Device(s)-Human Interactions}

Robots have long worked with other digital devices to accomplish tasks. The teleoperation of a robot requires signals and commands from other devices, such as screens $[1,10,17,33]$, controllers $[24,25]$ or even virtual reality $[18,40,44]$. Digital devices have also been used to supplement human-robot interactions. Walker et al. [41] used an augmented reality system to inform users about the status of a flying robot. Scassellati et al. [29] showed a system where an embodied robot's gaze was used to guide deaf infants to an avatar on a screen. These often are simultaneous interactions where users interact with multiple systems at the same time.

There has been little work exploring sequential interactions with multiple robots. Luria et al. [20] used a design research method of user-enactments $[9,22]$ to explore responses to agents that can move from one body to another to support a single service across touchpoints. Similar methods have been used to explore how agents might interact with groups of people [27]. Tan et al. [38] investigated how robots should interact with each other as they transfer users from one robot to another, and found that interaction between robots during person transfer is preferable to no interaction. Shiomi et al. [34] demonstrated a system in which a robot led customers to the front of the store and then transferred them to another robot who introduced the store. These studies were all context-specific 
and explored particular components of person transfers. Here, we propose a theoretical, generalizable taxonomy for the underlying concept of transfers.

\section{METHOD}

We held two sets of workshops with the goal of generating as many descriptions of transfer scenarios as possible. The workshops were approved by our university's institutional review board. We used a range of design ideation tools and methods to support this activity and inspire creativity. Throughout the workshops, we refined the tools and procedure to maximize the amount and quality of ideas.

\subsection{Scoping Workshop Procedure}

The scoping workshop consisted of three separate events with a total of 8 participants and was conducted in September and October, 2019. Each event had 2 or 3 participants and took about 2 hours. Participants were recruited through email lists and word of mouth, and they were compensated 20 USD for their participation. Participants were graduate students who studied robotics or human-computer interaction. We chose graduate students as they have high familiarity with current robot and device capabilities. After a few warm-up activities with Alternative Use and New Metaphors [19] exercises, participants were asked to generate ideas about how they envision multiple robots working together to provide services to people in the future. We chose not to limit ideation to sequential interactions in order to capture the natural occurrence of sequential interactions within the broader pool of ideas.

Each event consisted of multiple idea generation rounds. In each round, participants were given a specific location or context and provided with ideation cards. These were printed cards with words or images. In events 1 and 2, participants were given cards that specified the types of robots involved in an interaction, such as humanoid robot, flying robot, etc. Theses cards were removed in event 3. For events 2 and 3, participants were also given cards with prescribed relationship, e.g. sidekicks, swarms, or friends, to inspire new forms of interactions. After each round, participants shared and discussed some of their favorite ideas.

\subsection{Ideation Workshop Procedure}

The workshop was a single, 3-hour workshop with 7 designers as participants, and was conducted in early February, 2020. Participants were recruited through email lists and word of mouth and compensated 30 USD for their participation. We chose designers as they are accustomed to design processes and are capable of generating many high-quality ideas in a short amount of time. Furthermore, our goal did not require familiarity with special technologies. The workshop had two parts: ideation and idea clustering.

Ideation: After a warm-up exercise, participants took part in multiple rounds of idea generation. Similar to the scoping workshop, participants were shown a location or context in each round. Participants were given "Entity" cards labeled "Human", "Machine", or "Robot". The goal was to use these cards to define transfers between the selected entities. For example, participants were asked to draw 2 cards from the deck and generate ideas based on that combination. For example, a participant who drew "Human" followed by "Machine" would be asked to generate ideas involving a transfer from a human to a machine. Using custom cards allowed us to constrain some of the design space and encourage participants to creatively think of new transfer forms. Each round lasted about 5 minutes and was followed by a discussion that allowed participants to share ideas and inspire new ones.

Idea Clustering: Participants were split into three groups. Each group analyzed its own data and clustered it accordingly. This allowed participants to further elaborate on the different aspects of their ideas. Their clustering served as the inspiration and starting point for our analysis and taxonomy.

\subsection{Analysis}

For both workshops, the generated scenarios were analyzed by the research team using Affinity Diagramming [3]. We used this established method because we aimed to identify emerging themes rather than formulate a new theory. Instead of a coding scheme, this method relies on team members working together to reveal insights within the collected data [3]. Affinity diagramming was conducted iteratively through discussion over multiple sessions until the study team came to an agreement about the placement of all ideas. The clustering activity done by participants served as the starting point for our identification of important factors of sequential person transfers. This process was also supported by analysis of selected scenarios using FAST diagrams, which decompose services into their most basic actions by different entities [6].

\section{SCOPING WORKSHOP}

The goal of the scoping workshop was to understand the possible roles of robots in sequential interactions. In the three conducted sessions, participants generated a total of 132 ideas. Not all ideas were about transfers in interactions, which was our topic of interest.

As we chose not to limit workshop participants in their ideation of multi-robot interactions, the majority of ideas fell into the category of concurrent multi-robot interactions (e.g. "A physical service robot helps you go to [a] doctor's office while [another] robot carries your stuff.") or interactions where the two robots are part of the same entity (e.g. "[A robot] has a robotic arm attached [to it] to guide visitors \& point to exhibits while giving a tour"). Of the 132 generated ideas, we categorized $28(21 \%)$ of the ideas as sequential interactions. The imbalance in categories reflects the difficulty of envisioning interactions across time, and the current tendency to conceptualize multiple robot applications as swarms or simultaneous interactions.

\subsection{Findings}

Within the sequential human-multi-robot interactions generated by participants, we identified three categories.

Transfer of Product Between Robots In the first category, multiple robots transfer physical objects between them before engaging people. An example would be in food preparation. First, a robot in the kitchen prepares the food. Then, a second, mobile robot retrieves the food and delivers it to the customer. In these direct robot-to-robot transfers, the customer may not even be aware of the presence of the first robot. Alternatively, the customer might know about the interaction between the two robots but only directly interact with one of them. 
Transfer of User Information Between Robots For this category, information is transferred between robots, rather than physical objects. For example, a flying robot might detect that a customer wants a particular item on a high shelf in a store and direct a ground robot to come assist the customer and bring them the item. Here, the flying robot serves a backstage role and might never be seen by the customer, so they may not even realize that multiple robots were involved. Here, too, the user is likely to interface with just one robot.

Person Transfer Between Robots In this category, the user interacts first with one robot and then transferred to continue the interaction with another.

"[A robot] receives people at the counter for check-in, then directs [a] mobile robot to guide people to their respective waiting rooms." In the scenario above, the customer initially interacts with one robot and is then transferred to a mobile robot to complete the task. Both robots serve as user-facing robots.

\subsection{Discussion}

In both product and information transfers, a person might indirectly interact with the "backstage" robot. In the store example, the flying robot's role is solely to transfer information to the other robot when a customer needs assistance. Even if the person does not interact with the flying robot, they might notice that the interaction involved multiple robots. In these types of scenarios, it is worth considering whether these "backstage" robots should be visible or have any direct contact with people.

In contrast to product and information transfers, a person who is transferred will necessarily interact directly with multiple robots. This kind of transfer can happen under a variety of configurations that would influence how designers create the corresponding interactions. For example, a transfer where both robots are present in the same space as the person would be vastly different from a transfer in which the person needs to independently travel from a robot receptionist to a screen-based interface in another location.

Given the broad range of configurations, more insights were needed to analyze and understand the design and interaction nuances of person transfers with multiple robots. The few examples generated in the scoping workshop encouraged us to conduct the second design workshop with the goal of improving our understanding of this topic. Furthermore, as people today already undergo a variety of person transfers between human staff and digital systems, we intended to capture characteristics of such transfers in the second workshop, and learn how future human-robot interactions may map onto them.

\section{IDEATION WORKSHOP}

In the ideation workshop, participants generated 287 different "oneline" scenarios for a range of service contexts and domains (e.g., airports, medical centers). Given our goal to capture existing person transfers in services today in addition to imagining future situations, we asked participants to think about existing interactions and service transfers they are familiar with.

As participants were asked to generate quantity over quality (as is common in brainstorming activities), some ideas did not involve person transfers, but instead focused on the transfer of objects or information between systems and people (as in the previous workshop). For our analysis, we only included scenarios that fit the definition of Person Transfers Between Robots. Among the 287 generated ideas, 67 (23\%) fit this definition. These were combined with the 14 scenarios that match the definition from the scoping workshop for a total of 81 scenarios that we used in our analysis.

\subsection{Overview of Findings}

Through affinity diagramming, we created a taxonomy for person transfers between robots. An overview of the taxonomy is shown in Figure 1. The taxonomy includes four dimensions:

Type of Person Transfers The service journey touchpoints where person transfers occur.

Rationale behind Person Transfer The interaction and service needs that drive the inclusion of transfers in the service.

Design of Person Transfers The implementation variables of the transfer throughout the service (time, space, and form).

Information Shared The different information shared and used between entities in person transfers.

While some categories within these four dimensions are not mutually exclusive, considering each separately can contribute to a fuller understanding of person transfers and how to design for them. Rather than a comprehensive taxonomy, we present a set of prominent dimensions that should be considered for person transfers. We also included scenarios from the workshops (italicized and annotated with "”) to help motivate each category.

\subsection{Types of Person Transfers}

The first dimension looks at the moment in the person's service experience in which they experience a person transfer. The transfer can be either planned or unplanned. A planned person transfer is a transfer that always occurs and is required as part of the service experience. An unplanned transfer only occurs in some cases, deviates from the normal interaction, and is not intended to be experienced by the majority of people.

5.2.1 Planned Person Transfer. A planned person transfer can be further separated into a Person Transfer between Tasks and a Person Transfer within a Task. The distinction between tasks and subtasks is often ambiguous. For example, the experience of "getting on a flight" can be further decomposed into sub-tasks such as checking in and going through security. Each sub-task can be further subdivided into smaller actions; e.g., going through security may involve moving between the luggage scanner and human security staff. For our taxonomy, we define a task as a collection of compulsory actions that are mandatory to complete. In the airport security example, the person will fail the task if they choose to only use the luggage scanner but not the person scanner.

Person Transfer within a Task These are scenarios where person transfers are an integral part of the interaction, and connect two actions within the same task.

"You print your boarding pass and luggage tag, then place [your] luggage on the conveyor belt."

In this case, if the person wants to drop off their luggage, they need to transfer between different systems to complete the task. 


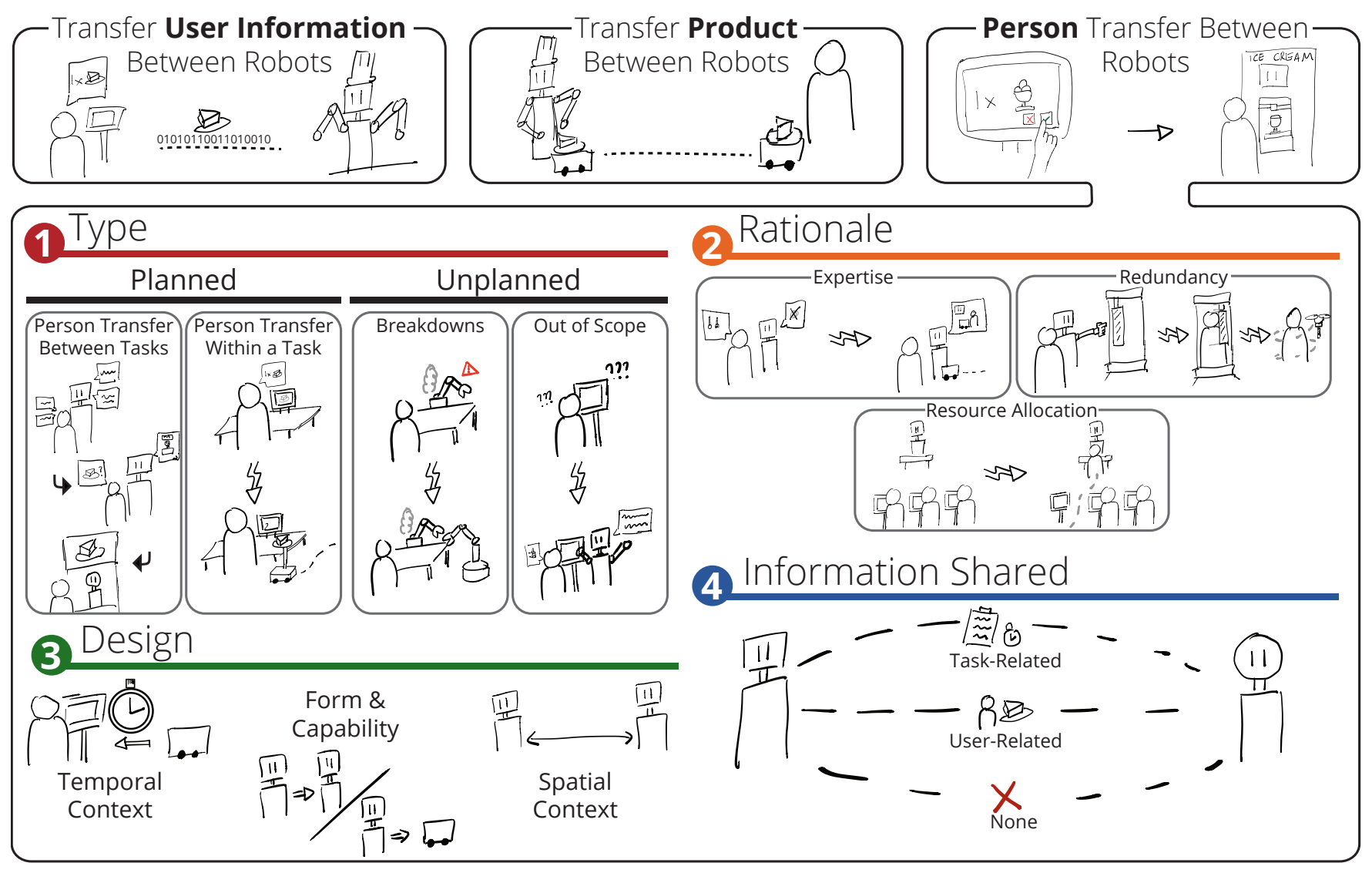

Figure 1: Overview of the taxonomy.

Person Transfer between Tasks This category describes situations in which people might do two different tasks in sequenceeach task is independent, and the transfer only occurs if the person would like to engage in both parts of a service. This is likely to happen as part of a bundled service where different but complementary services are combined to increase customer convenience and value [2].

"Patient receives physical therapy before acupuncture treatment in the same center."

While both treatments are part of the same service, they are not dependent and can be completed separately and in any order.

"A person checks in electronically and is directed to a waiting room, then [they are] summoned by a screen."

Transfers between tasks also include scenarios where people are transferred to a waiting area or given a notification device. We consider the waiting as a separate task, as it can potentially be skipped or even avoided if the service is optimized.

5.2.2 Unplanned Person Transfers. Unplanned person transfers are person transfers that are not intended to be part of the standard user experience. We identified two types:

Breakdowns \& Failures These are person transfers caused by machine malfunction or human limitations, which may prevent them from being able to continue the task.
"A ticket machine breaks down, [it] calls over maintenance." These breakdowns are not limited to machines-a human staff member may encounter challenges that prevent them from completing a task as well. This category also includes scenarios where the intended interaction fails due to extraneous circumstances.

"A tour guide cannot be heard by all tour members, so they refer tour members to an audio guide."

In this example, the tour group is too large for everyone to be able to hear the guide, resulting in a transfer from the tour guide to audio guides.

Out of Scope The second reason for unplanned transfers can be that a requested service is not part of the first robot's job scope or capabilities. While these transfers are likely to be pre-planned by the service, they are not requested by all users and often serve as a catch-all for any situation that robots might not understand or be able to address.

"[You] see the menu on an iPad, [then you] ask for clarification about ingredients [from] a waiter."

The device lacks information about the person's intent, which may force them to transfer the interaction to another entity (a human employee, in this case) to retrieve the needed information.

"You use an ATM to take out cash and then ask a bartender to break the large bills." 
Similarly, the customer transfers from the machine to a human staff due to the machine's inability to complete the task.

\subsection{Rationale Behind Person Transfer}

The second dimension looks at the reasons why incorporating person transfers may be valuable to a service.

Expertise In some instances, the first and second entities have different capabilities, both of which are required to complete the task. For example, the first entity that interacts with a person may be a good "user-facing" entity but lack the capability to fulfill their request, requiring it to rely on another entity.

"Tablets stationed for people to enter where they want to go, [for a robot] to guide them."

The tablet lacks the ability to move and guide users, therefore it would need to rely on the mobile robot to complete any navigational guidance tasks.

Resource Allocation Using multiple entities may better allocate a service's resources and allow more people to receive service simultaneously. Instead of using a single entity, a service can be split to smaller parts and assigned to separate entities, similar to an "assembly line". Breaking down a task also allows some entities to engage "when needed"-they do not need to pay attention to every customer at every given moment.

"At food courts: tablet to order food, mobile robot delivers."

In this example, multiple people can order food simultaneously through the tablet and only a few robots deliver all of the food when it is ready.

Redundancy Multiple entities take part in the same task to ensure safety by cross-checking or serving as a backup.

"Metal detectors [do] the first pass at the front of the museum, then someone goes through your bag."

Here, the person interacts with two entities, both of which exist for the same goal of detecting forbidden objects. Repeated interactions with several entities help mitigate potential errors.

\subsection{Design of Person Transfers}

The third dimension in the taxonomy is how person transfers are implemented and what actions a service provider might expect from people. This dimension covers both the physical and interactional aspects of person transfers.

5.4.1 Spatial Context. A person transfer can happen in a variety of spatial contexts. For example, both entities could be co-located, or they may never be co-present and thus require the person to move independently to complete the interaction (remote).

Co-Located These are person transfers that occur when both entities are present in the same space as the person.

"[A robot] receives people at the counter for check-in, then directs a mobile robot to guide people to their respective waiting rooms." The person interacts with the first robot, who then summons the mobile robot. At one point in the interaction (after the mobile robot appears), both robots are co-located in a single place. This allows both robots to interact with the person and each other.

Remote The alternative is to have two entities in different locations, which requires the person to move from one location to another to complete the task.
"Ticket machine directs visitors to a navigation computer." From the person's point of view, there is no visible interaction between the entities. Furthermore, the interaction may differ based on how remote the entities are from each other. For example, they can be in view of each other, or they can be located farther apart in different rooms, requiring more instructions on how to locate the other entity.

5.4.2 Temporal Context. In addition to location changes, a temporal gap may also exist during person transfers. This gap can be caused either by the time needed for the second entity to join the interaction or by the time needed for the person to reach the second entity. Similar to spatial contexts, timing can also range from occurring immediately after the initiation of a transfer to occurring after some time gap between the two interactions.

Immediate These are situations where the second encounter happens nearly instantaneously after the first.

"Bouncer checks [you] before you go in, machine scans ID [and] unlocks door."

This kind of interaction is often coupled with co-located entities. Gaps Alternatively, transfers might take a while, as the person moves to the second entity or as they wait for the second part of the interaction.

"Bartender asks you about what kind of drink you feel like getting, then directs you to a cocktail machine where you do it yourself." The need to travel to the next machine creates a gap in time.

"Ordering food via a screen on flight. Flight attendant has to bring it to you."

Some gaps can also be caused by the time needed for the second entity to arrive after the person initiates the request.

5.4.3 Form \& Capability. As this is an exploration of transfers based on existing interactions between robots, devices, and human staff members, entities can take on different forms with vastly different capabilities. A person might be transferred between two entities with a similar form (e.g., human to human) or different forms (e.g., human to robot). The form is not a simple binary category, but instead a spectrum of similarity. Two humans wearing similar uniforms will have more similarity than two humans who are not. This is important in transfers because visual similarity provides some indication about with whom people should interact next. These cues can be designed for non-human entities, for example by using comparable branding or color palettes.

Form is also important because it shapes the entity's perceived capabilities [12,36] and trustworthiness [30]. A robot with a visible face and mouth is more likely to be perceived as able to verbally communicate compared to a flying drone. The degree of difference between forms in person transfers can alter the amount of introduction and explanation that is required as part of the interaction.

\subsection{Information Shared}

The final category in our taxonomy regards the information that is being shared between entities during the process of person transfers. As digital systems have the ability to transfer and process large amounts of information, they can easily share information with the next system when person transfers occur. We identified three types of information sharing: 
None Information sharing does not always happen during person transfers. In some cases, the person might be asked to re-convey the information to the second entity to complete the task.

"Audio guide tells the visitor to ask a docent for additional information."

In this situation, it is likely that the docent will not be aware of the visitor's intent and will require them to explain their needs.

User-Related The second type sets out to transfer newly learned information from the user, for example, what they hope to achieve.

"[There is an] iPad on a restaurant table for ordering food and calling staff to deliver the check and pay."

The patron communicates their intent through an iPad-the staff is likely to be aware of their intent upon arrival and already have their bill ready to go. This can also include non-task related personal information that the person disclose.

Task-Related The most common information shared in our findings is regarding the state of the task. Task information about what had happened is generated by the first entity and used to inform the next entity and help its decision process. This information could be as simple as audio notifications or as complex as information about the state of the task.

"[An] X-ray security machine beeps so [the] human has to do a pat down."

"Self checkout machine where you get your blood pressure. It talks to you to say "I sent your information to the nurse, she will come in a minute."”

Furthermore, the sharing can be bidirectional, e.g., the second entity can send back information on their status and arrival time.

\section{UTILIZING THE TAXONOMY}

In this section, we detail ways in which our taxonomy can be used for interaction design and research.

\subsection{Interaction Design}

To highlight the potential of our taxonomy for interaction design, we position it within a hypothetical design scenario-the design process for Tri-bot, an emergency room triage robot that helps with basic tasks such as measuring patients' vitals, interviewing them about their conditions, and leading them to different examination rooms accordingly. Besides its primary tasks of escorting and collecting patient's vitals and information, Tri-bot has to receive and transfer patients to other humans and machines for various treatments. We discuss how our taxonomy may be of use to Tri-bot's designers and to the hospital in various stages of the design process. Need finding: Using the taxonomy, we can identify the primary role the robot should play. We can also use vocabulary provided by the taxonomy to help designers and stakeholders on the team discuss the different interactions between the robot and the users, and the range of transfers it would need to make.

Tri-bot's primary role is to help with resource allocation by taking on basic tasks and allowing nurses to focus on more critical tasks. Most of Tri-bot's transfers will happen within a task, as the patients move between healthcare professionals.

Robot behavior design: The taxonomy can help designers plan and design the robot's behavior during transfers, depending on the transfer type and the different aspects it involves.
As patients are led around the hospital, nearly all transfers will be co-located. However, these transfers can happen immediately or after a (temporal gap), depending on the availability of machines and clinicians. Therefore, Tri-bot needs to adapt its response based on the duration of the temporal gap and perhaps provide additional attention if the gap is too long.

Physical design: The design of the robot's form and capabilities can also be informed by the types of transfers it will need to perform (identified in the previous two phases).

The hospital may want Tri-bot to serve as a bridge between the initial triage nurse and other healthcare team members. In the beginning, Tri-bot's physical appearance is different from a typical nurse, and it is likely that a new patient will not have a mental model of Tri-bot's role. This will require introductions and guides for patients. Throughout the service experience, patients are likely to interact with similar Tri-bots and become familiar with how they look and work. Over time, less introduction and explanation will be needed.

Inter-System Connection: The taxonomy can also assist in reflecting on what kind of information will be shared across robots and other service entities within a service. Designers should also determine how to disclose and be transparent about the information that is being shared.

As a task-driven robot, Tri-bot is most likely to share task-related information with other team members. Patients are likely to expect health information and vitals to be shared with their doctors; therefore, these transfers will not require patient consent. However, the service provider needs to determine how much user-related information is shared between systems. For example, making use of personal information disclosed during prior interactions might improve rapport with the patient, but it could also be off-putting.

\subsection{Researchers}

In addition to designers, our taxonomy can also be useful for researchers in various methodologies. For example, it could be used during field work and observations to categorize and describe person transfers. An observation report may note:

The patient was transferred between tasks when they were directed to the checkout machine. The transfer was remote because the patient was given directions to go to the checkout desk by Tri-bot. Unfortunately, they got lost because the robot failed to....

For evaluative user studies, the taxonomy can be used to isolate specific factors of person transfers to be further studied. For instance, a researcher interested in the effect of spatial context on the perceptions of robots could use this taxonomy to ensure other related factors are kept constant in their study.

This research explored how spatial and temporal contexts in a user transfer scenario affect user experience. In this $2 x 2$ user study, we ....

We can also analyze prior work according to this taxonomy. For instance, in [38], researchers conducted a study exploring how different interactions between robots with different forms affect robot perception in a co-located, immediate, and within-task setting. In [34], researchers demonstrated a co-located, within-task person transfer of two field robots with the same form. In [16], researchers 
showed a system that determines whether a user undergoes a colocated or remote person transfer based on efficiency.

Finally, this taxonomy has identified under-explored aspects of person transfers, thereby revealing high-priority research needs in these less known but important areas. Some examples include:

Types How does transfer type influence the information shared? How can we improve seamless transfers without sacrificing privacy?

Rationale What are the downsides of person transfers in different contexts, compared to using a single agent?

Design What are the trade-offs between different design choices, and how can interactions mitigate them? How can remote person transfers be better supported through design?

Information How should entities handle privacy and informational disclosure during person transfers? How might users perceive sharing of user-related information?

\section{DISCUSSION}

\subsection{Transfer Trade-Offs}

Sequential person transfers are beneficial in that they can create a more efficient service flow. They allow services to widely distribute resources to engage many people simultaneously, and they can utilize robots that are designed for a specific job. However, the decision to include person transfers is often a result of trade-offs among different factors. For instance, in a human guidance scenario, using the same robot for both kiosk-type services and guidance services will be faster as there is no time spent on a transfer. Furthermore, transfers would require additional logic and interaction complexity that must be explained and understood by people, which increases the odds of technological mistakes and failures. On the other hand, splitting the interaction might be more cost-effective because the service could use simpler, specialized robots for different parts of the task and serve multiple people. Thus, designers and service providers should consider whether the efficiency and service flow that person transfers between multiple robots create are important enough to outweigh the problems of person transfers: development cost, user misunderstandings, and potential technological error.

\subsection{Complexity in Transfer Interaction Design}

If a service decides to undertake person transfers, several aspects that the taxonomy points out need to be considered: How far one robot is from the other (spatial context), how much waiting time might arise between the first robot and the second robot (temporal context), and how does each robot look (form).

A temporal gap between the two interactions with robots can reduce the quality of the experience. Designers need to consider how to engage or support a person during the gap. Similarly, large spatial gaps require ways to convey directions and additional support for lost users. The larger the gap, the more difficult it will be to create a seamless flow from one interaction to the other, and the less likely it will be for the interaction to be perceived as a single, holistic one.

Finally, the form of the robots can also significantly impact the interaction and the potential success of a transfer. Any transfer between two robots with different shapes or exterior designs will require some visual cue to communicate that they are part of the same interaction. The less similar the two robots are, the more a transfer entity will need to clearly communicate the role of each robot and information shared among them. Similar forms can also be achieved through robot re-embodiment, where the robot's "personality" or "identity" transitions from one robot body to another and indicates to the user that a transition has occurred $[15,20]$.

\section{LIMITATIONS}

Our design workshops primarily focused on service contexts where people are the customers. While we attempted to cover a large variety of contexts, such as healthcare, transportation, etc., other contexts might present unique characteristics and alternative needs for person transfers. Furthermore, our work did not touch on nonservice environments, such as the home and workplace. Those spaces present additional challenges and considerations, such as robots' dynamics with different members of the family. The workshops also only explored scenarios where a second entity was available. This underlying assumption may not always be valid. There will be scenarios where a second robot might not always be available. Failed transfers should also be further explored.

One of the challenges of this work was that the phrase "person transfers" is somewhat ambiguous, and could mean a variety of things to participants based on their personal interpretation. Participants mostly understood "person transfers" as a sequential action where something moves from one entity to another, but they occasionally included ideas outside of this scope.

Lastly, we chose to be flexible with the interpretation of our scenarios as the categorization of each scenario was loosely defined. For example, in the scenario "iPad on restaurant table for ordering food and calling staff to deliver check and pay", if we added "elsewhere", the transfer could change from a co-located spatial transfer to a remote transfer. Thus, we extracted insightful categories, even if they were clearly represented in only one or two scenarios in our collected scenarios. This was done in order to obtain a broad understanding of the factors that might impact this design space.

\section{CONCLUSION}

As robotic systems are being deployed in parts of a service, they need to work with other devices, robots, and even human staff. Our taxonomy provides a blueprint for researchers and designers to evaluate different person transfers and potential opportunities in the area. The four primary dimensions of the taxonomy-Rationale, Type, Design, and Information Shared-help categorize and make sense of the factors that impact person transfers between robots and other entities. We discuss potential opportunities and tradeoffs in including person transfers across the different categories. Through a better understanding of the dimensions of person transfers, it is possible to design better experiences that integrate diverse technological devices into a seamless service experience.

\section{ACKNOWLEDGMENT}

This work was funded by the National Science Foundation (SES1734456) and the National Institute on Disability, Independent Living, and Rehabilitation Research (90DPGE0003). We also thank Samantha Reig, Elizabeth Carter, and our workshop participants. 


\section{REFERENCES}

[1] Salvatore Andolina and Jodi Forlizzi. 2015. A multi-touch interface for multi-robot path planning and control. In International Workshop on Symbiotic Interaction. Springer, 127-132.

[2] Leonard L Berry and Sandra K Lampo. 2000. Teaching an old service new tricks: The promise of service redesign. Fournal of Service Research 2, 3 (2000), 265-275.

[3] Hugh Beyer and Karen Holtzblatt. 1997. Contextual design: A customer-centered approach to systems designs.

[4] Mary Jo Bitner, Amy L Ostrom, and Felicia N Morgan. 2008. Service blueprinting a practical technique for service innovation. California management review 50, 3 (2008), 66-94

[5] Frederik Brudy, Christian Holz, Roman Rädle, Chi-Jui Wu, Steven Houben, Clemens Nylandsted Klokmose, and Nicolai Marquardt. 2019. Cross-Device Taxonomy: Survey, Opportunities and Challenges of Interactions Spanning Across Multiple Devices. In Proceedings of the 2019 CHI Conference on Human Factors in Computing Systems. ACM, 562.

[6] Charles W Bytheway. 2007. FAST creativity and innovation: Rapidly improving processes, product development and solving complex problems. J. Ross Publishing.

[7] Xiang 'Anthony' Chen, Tovi Grossman, Daniel J. Wigdor, and George Fitzmaurice. 2014. Duet: Exploring Joint Interactions on a Smart Phone and a Smart Watch In Proceedings of the SIGCHI Conference on Human Factors in Computing Systems (Toronto, Ontario, Canada) (CHI '14). Association for Computing Machinery, New York, NY, USA, 159-168. https://doi.org/10.1145/2556288.2556955

[8] Jorgos Coenen, Niels Wouters, and Andrew Vande Moere. 2016. Synchronized wayfinding on multiple consecutively situated public displays. In Proceedings of the 5th ACM International Symposium on Pervasive Displays. 182-196.

[9] Scott Davidoff, Min Kyung Lee, Anind K Dey, and John Zimmerman. 2007. Rapidly exploring application design through speed dating. In International Conference on Ubiquitous Computing. Springer, 429-446.

[10] Terrence Fong, Charles Thorpe, and Charles Baur. 2003. Multi-robot remote driving with collaborative control. IEEE Transactions on Industrial Electronics 50 , 4 (2003), 699-704

[11] Giuseppe Ghiani, Fabio Paternò, and Carmen Santoro. 2010. On-demand crossdevice interface components migration. In Proceedings of the 12th international conference on Human computer interaction with mobile devices and services. ACM, 299-308.

[12] Jennifer Goetz, Sara Kiesler, and Aaron Powers. 2003. Matching robot appearance and behavior to tasks to improve human-robot cooperation. In The 12th IEEE International Workshop on Robot and Human Interactive Communication, 2003. Proceedings. ROMAN 2003. Ieee, 55-60.

[13] Tim Hornyak. 2020. Meet the robots that may be coming to an airport near you CNBC (2020). https://www.cnbc.com/2020/01/10/meet-the-robots-that-may-becoming-to-an-airport-near-you.html

[14] Tharon Howard. 2014. Journey mapping: A brief overview. Communication Design Quarterly Review 2, 3 (2014), 10-13.

[15] Michita Imai, Tetsuo Ono, and Tameyuki Etani. 1999. Agent migration: communications between a human and robot. In IEEE SMC'99 Conference Proceedings. 1999 IEEE International Conference on Systems, Man, and Cybernetics (Cat. No. 99CH37028), Vol. 4. IEEE, 1044-1048.

[16] Piyush Khandelwal and Peter Stone. 2017. Multi-robot human guidance: Human experiments and multiple concurrent requests. In Proceedings of the 16th Conference on Autonomous Agents and MultiAgent Systems. International Foundation for Autonomous Agents and Multiagent Systems, 1369-1377.

[17] Kexi Liu, Daisuke Sakamoto, Masahiko Inami, and Takeo Igarashi. 2011. Roboshop: multi-layered sketching interface for robot housework assignment and management. In Proceedings of the SIGCHI Conference on Human Factors in Computing Systems. ACM, 647-656.

[18] Oliver Liu, Daniel Rakita, Bilge Mutlu, and Michael Gleicher. 2017. Understanding human-robot interaction in virtual reality. In 2017 26th IEEE International Symposium on Robot and Human Interactive Communication (RO-MAN). IEEE, 751-757.

[19] Dan Lockton, Devika Singh, Saloni Sabnis, Michelle Chou, Sarah Foley, and Alejandro Pantoja. 2019. New Metaphors: A Workshop Method for Generating Ideas and Reframing Problems in Design and Beyond. In Proceedings of the 2019 on Creativity and Cognition (San Diego, CA, USA) (C\&\#38;C '19). ACM, New York, NY, USA, 319-332. https://doi.org/10.1145/3325480.3326570

[20] Michal Luria, Samantha Reig, Xiang Zhi Tan, Aaron Steinfeld, Jodi Forlizzi, and John Zimmerman. 2019. Re-Embodiment and Co-Embodiment: Exploration of social presence for robots and conversational agents. In Proceedings of the 2019 on Designing Interactive Systems Conference. ACM, 633-644.

[21] Nicolai Marquardt, Ken Hinckley, and Saul Greenberg. 2012. Cross-Device Interaction via Micro-Mobility and f-Formations. In Proceedings of the 25th Annual ACM Symposium on User Interface Software and Technology (Cambridge, Massachusetts, USA) (UIST '12). Association for Computing Machinery, New York, NY, USA, 13-22. https://doi.org/10.1145/2380116.2380121

[22] William Odom, John Zimmerman, Scott Davidoff, Jodi Forlizzi, Anind K Dey, and Min Kyung Lee. 2012. A fieldwork of the future with user enactments. In Proceedings of the Designing Interactive Systems Conference. 338-347.
[23] Jeffrey S. Pierce and Jeffrey Nichols. 2008. An Infrastructure for Extending Applications' User Experiences across Multiple Personal Devices. In Proceedings of the 21st Annual ACM Symposium on User Interface Software and Technology (Monterey, CA, USA) (UIST '08). Association for Computing Machinery, New York, NY, USA, 101-110. https://doi.org/10.1145/1449715.1449733

[24] Pragathi Praveena, Guru Subramani, Bilge Mutlu, and Michael Gleicher. 2019. Characterizing Input Methods for Human-to-robot Demonstrations. In 2019 14th ACM/IEEE International Conference on Human-Robot Interaction (HRI). IEEE, 344353

[25] Daniel Rakita, Bilge Mutlu, and Michael Gleicher. 2017. A motion retargeting method for effective mimicry-based teleoperation of robot arms. In Proceedings of the 2017 ACM/IEEE International Conference on Human-Robot Interaction. ACM, 361-370.

[26] Dimitrios Raptis, Jesper Kjeldskov, and Mikael B Skov. 2016. Continuity in multidevice interaction: an online study. In Proceedings of the 9th Nordic Conference on Human-Computer Interaction. ACM, 29.

[27] Samantha Reig, Michal Luria, Janet Z Wang, Danielle Oltman, Elizabeth Jeanne Carter, Aaron Steinfeld, Jodi Forlizzi, and John Zimmerman. 2020. Not Some Random Agent: Multi-person interaction with a personalizing service robot. In Proceedings of the 2020 ACM/IEEE International Conference on Human-Robot Interaction. 289-297.

[28] Stephanie Santosa and Daniel Wigdor. 2013. A field study of multi-device workflows in distributed workspaces. In Proceedings of the 2013 ACM international joint conference on Pervasive and ubiquitous computing. ACM, 63-72.

[29] Brian Scassellati, Jake Brawer, Katherine Tsui, Setareh Nasihati Gilani, Melissa Malzkuhn, Barbara Manini, Adam Stone, Geo Kartheiser, Arcangelo Merla, Ari Shapiro, et al. 2018. Teaching language to deaf infants with a robot and a virtual human. In Proceedings of the 2018 CHI Conference on Human Factors in Computing Systems. ACM, 553.

[30] Kristin E Schaefer, Tracy L Sanders, Ryan E Yordon, Deborah R Billings, and Peter A Hancock. 2012. Classification of robot form: Factors predicting perceived trustworthiness. In Proceedings of the human factors and ergonomics society annual meeting, Vol. 56. SAGE Publications Sage CA: Los Angeles, CA, 1548-1552.

[31] Christopher Sebes. 2020. Restaurant Reopenings Hold Promise For Certain Operational Models And Technologies. Forbes (2020) https://www.forbes.com/sites/forbestechcouncil/2020/07/10/restaurantreopenings-hold-promise-for-certain-operational-models-and-technologies

[32] Hamza Shaban. 2020. Amazon launches new grocery store with 'smart' shopping carts and Alexa guides. The Washington Post (2020). https://www.washingtonpost. com/business/2020/08/27/amazon-fresh-store-los-angeles/

[33] Meher T Shaikh and Michael A Goodrich. 2017. Design and evaluation of adverb palette: A gui for selecting tradeoffs in multi-objective optimization problems. In 2017 12th ACM/IEEE International Conference on Human-Robot Interaction (HRI. IEEE, 389-397.

[34] Masahiro Shiomi, Takayuki Kanda, Dylan F Glas, Satoru Satake, Hiroshi Ishiguro, and Norihiro Hagita. 2009. Field trial of networked social robots in a shopping mall. In 2009 IEEE/RSf International Conference on Intelligent Robots and Systems. IEEE, 2846-2853.

[35] G Lynn Shostack. 1982. How to design a service. European journal of Marketing (1982).

[36] Valerie K Sims, Matthew G Chin, David J Sushil, Daniel J Barber, Tatiana Ballion, Bryan R Clark, Keith A Garfield, Michael J Dolezal, Randall Shumaker, and Neal Finkelstein. 2005. Anthropomorphism of robotic forms: A response to affordances?. In Proceedings of the Human Factors and Ergonomics Society Annual Meeting, Vol. 49. SAGE Publications Sage CA: Los Angeles, CA, 602-605.

[37] Xiang Zhi Tan, Michal Luria, and Aaron Steinfeld. 2020. Defining Transfers Between Multiple Service Robots. In Companion of the 2020 ACM/IEEE International Conference on Human-Robot Interaction (Cambridge, United Kingdom) (HRI '20). Association for Computing Machinery, New York, NY, USA, 465-467. https://doi.org/10.1145/3371382.3378258

[38] Xiang Zhi Tan, Samantha Reig, Elizabeth J Carter, and Aaron Steinfeld. 2019. From One to Another: How Robot-Robot Interaction Affects Users' Perceptions Following a Transition Between Robots. In 2019 14th ACM/IEEE International Conference on Human-Robot Interaction (HRI). IEEE, 114-122.

[39] Meg Tonkin, Jonathan Vitale, Sarita Herse, Mary-Anne Williams, William Judge, and Xun Wang. 2018. Design methodology for the ux of hri: A field study of a commercial social robot at an airport. In Proceedings of the 2018 ACM/IEEE International Conference on Human-Robot Interaction. 407-415.

[40] Nhan Tran, Josh Rands, and Tom Williams. 2018. A hands-free virtual-reality teleoperation interface for wizard-of-oz control. In Proceedings of the 1st International Workshop on Virtual, Augmented, and Mixed Reality for HRI (VAM-HRI).

[41] Michael Walker, Hooman Hedayati, Jennifer Lee, and Daniel Szafir. 2018. Communicating robot motion intent with augmented reality. In Proceedings of the 2018 ACM/IEEE International Conference on Human-Robot Interaction. ACM, 316-324.

[42] Mark Weiser. 1991. The Computer for the 21 st Century. Scientific american 265, 3 (1991), 94-105.

[43] Mark Weiser and John Seely Brown. 1996. Designing calm technology. PowerGrid fournal 1, 1 (1996), 75-85. 
[44] David Whitney, Eric Rosen, Daniel Ullman, Elizabeth Phillips, and Stefanie Tellex 2018. Ros reality: A virtual reality framework using consumer-grade hardware for ros-enabled robots. In 2018 IEEE/RSf International Conference on Intelligent Robots and Systems (IROS). IEEE, 1-9.
[45] Daisy Yoo, John Zimmerman, Aaron Steinfeld, and Anthony Tomasic. 2010. Understanding the space for co-design in riders' interactions with a transit service. In Proceedings of the SIGCHI Conference on Human Factors in Computing Systems. 1797-1806. 